\title{
Analysis of Probabilistic Roadmaps for Path Planning
}

\author{
Lydia E. Kavraki* Mihail N. Kolountzakis ${ }^{\dagger} \quad$ Jean-Claude Latombe ${ }^{\ddagger}$
}

\begin{abstract}
We provide an analysis of a recent path planning method which uses probabilistic roadmaps. This method has proven very successful in practice, but the theoretical understanding of its performance is still limited. Assuming that a path $\gamma$ exists between two configurations $a$ and $b$ of the robot, we study the dependence of the failure probability to connect $a$ and $b$ on (i) the length of $\gamma$, (ii) the distance function of $\gamma$ from the obstacles, and (iii) the number of nodes $N$ of the probabilistic roadmap constructed. Importantly, our results do not depend strongly on local irregularities of the configuration space, as was the case with previous analysis. These results are illustrated with a simple but illuminating example. In this example, we provide estimates for $N$, the principal parameter of the method, in order to achieve failure probability within prescribed bounds. We also compare, through this example, the different approaches to the analysis of the planning method.
\end{abstract}

Keywords: Robot Path Planning, Randomized Algorithms, Probabilistic Roadmaps.

${ }^{*}$ Rice University, Department of Computer Science MS 132, Houston, TX 77005. This work was done while the author was with the Robotics Laboratory, Department of Computer Science Stanford University, Stanford, CA 94305 .

${ }^{\dagger}$ Department of Mathematics, University of Illinois at Urbana-Champaign, Urbana, IL 61801. This work was done while the author was with the School of Mathematics, Institute of Advanced Study, Princeton, NJ 08540.

$\ddagger$ Robotics Laboratory, Department of Computer Science Stanford University, Stanford, CA 94305. 

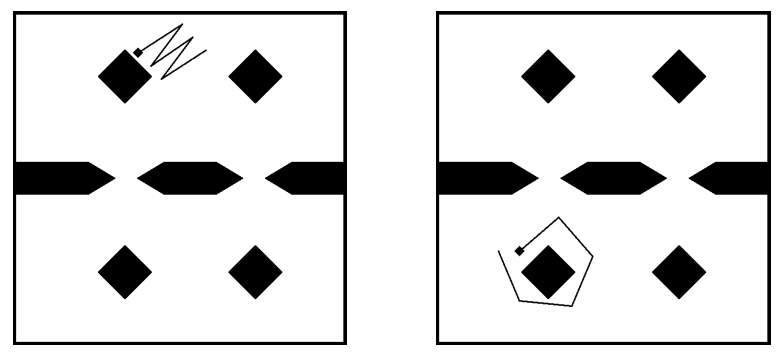

(a)
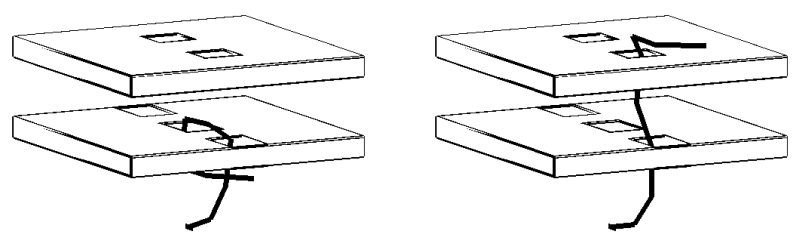

(b)

Figure 1: Examples of motion path problems solved by the probabilistic method

\section{Introduction}

Motion planning has been an active area of research during the last two decades [18]. The problem has gained increasing attention because of the larger number of potential applications (e.g. robotics, manufacturing, computer-assisted surgery, molecular biology). Several recent papers describe path planners that can deal with robots that have more than 3 or 4 degrees of freedom (dof) and move in realistic environments (for a survey see [12]). Because of the high computational complexity of path planning, practical planners employ different kinds of heuristics to guide the search of the robot from its initial to its final position $[1,2,3,5,8,9,10,11$, $13,16,20,21]$.

This paper considers the success of a class of probabilistic algorithms for path planning [11, $12,13,14,20,21]$, and tries to establish a framework for the theoretical understanding of their results. Our ultimate goal is to further enhance the performance of these methods by evaluating the role of their parameters and by finding ways to choose good values for these parameters. We will restrict ourselves to the description of the planner in $[12,13,14]$ for a concise presentation of the algorithm and our results. We hereafter refer to this planner as PRM (Probabilistic RoadMap planner). 
PRM builds probabilistic roadmaps in the free configuration space (C-space) [19] of the robot by generating and interconnecting a large number of (mostly) random configurations of the robot. These roadmaps are used for answering path planning queries: given an initial and final configuration of the robot, PRM connects them to the roadmap by simple paths (e.g. straight segments) and then searches the roadmap for a sequence of edges from one connecting node to the other. Concatenation of the relevant local paths produces an answer to the user query. PRM has been applied with excellent results to free flying and articulated robots moving in the plane or in space, as well as to non-holonomic robots. Examples of its capabilities are given in Figure 1. The robot in Figure 1(a) has 7 dof. PRM answers path planning queries, like the one defined by the configurations in Figure 1(a), in a fraction of a second after 50 seconds of preprocessing time on a DEC Alpha workstation rated at 126.0 SPECfp92 and 74.3 SPECint92. For similar query times, 620 seconds are spent in the preprocessing stage for the robot of Figure 1(b) which has 16 dof. Recently PRM is being applied to examples from assembly maintainability similar to the ones in [6] (aircraft engines).

As described in $[12,13,14]$ PRM requires the tuning of several parameters which depend on the considered workspace and robot. For example, one such parameter is $N$, the size of the network that sufficiently captures the connectivity of the free C-space within a given probability. Currently, the output roadmap is augmented by generating more nodes until the given initial and final configuration of the robot get connected through it. The theoretical estimation of $N$ can make the full automation of the technique possible, and permit its application in a wide variety of environments with minimal user effort.

The theoretical analysis of PRM is a difficult task. The work in [15] initiated the analysis. It related the performance of the planner to the goodness of the C-space of the problem in consideration. A space $S$ is called $\epsilon$-good if the volume of $S$ that each point in $S$ can "see" is at least a $\epsilon$ fraction of the total free volume of $S$. In the PRM framework, a point sees another point if it can be connected to it in a simple way (e.g. by a straight-line path). With the above definition, the value of $\epsilon$ is constrained by the point of the space which sees the least volume of $S$, which may be very small. Using $\epsilon$ we can estimate how many nodes a roadmap needs to have, so that the roadmap itself can see most of the C-space with high probability, and thus answer planning queries correctly with high probability.

The analysis in this paper focuses on understanding how the properties of the space in which 
the robot moves, the shape of the robot, and the features of the possible paths among its distinct configurations influence parameters of the technique such as the size of the roadmaps that must be produced by preprocessing. We adopt the following point of view. Assuming that a path between two different configurations of the robot exists, we show that the probability of failure to connect these configurations with PRM depends on (i) the length of the assumed path, (ii) the distance of the path from the obstacles, and (iii) the number of nodes of the roadmap generated. Using our results and making simple assumptions for the values of (i) and (ii) above, as well as for the failure probability we are willing to tolerate, we can estimate the size (number of nodes) of the probabilistic roadmap that finds the assumed path with the given probability. Or, if the shortest path between two configurations is known in a given environment, we can estimate the size of the roadmap that will permit us to find a path which is $\eta$-close to the shortest path. Our analysis is not very sensitive to local difficulties of the $\mathrm{C}$-space and carries over in any $\mathrm{C}$-space dimension.

The analysis given in this paper together with the analysis in [15] are also presented in the context of the general planning scheme in [4]. In that work the distance of the robot from the obstacles in the workspace is used to define a random sampling scheme for path planning. PRM can be regarded as an instance the sampling scheme in [4]. Before proceeding let us also mention that the theoretical evaluation of algorithms that are experimentally successful in path planning has recently attracted considerable attention. Some examples of research in this direction can be found in $[1,7,17]$. The work in [1] includes a proof of the probabilistic completeness of the algorithm presented in $[1,5]$, while the work in [17] discusses the performance of [3]. A learning algorithm for planning is presented and analyzed in [7].

\section{Description of PRM}

\subsection{PRM in Practice}

Practical implementations of PRM $([12,13,14])$ consist of a preprocessing and a query phase. Preprocessing is further divided into the roadmap construction and the roadmap enhancement steps.

During roadmap construction, random configurations of the robot (nodes) are generated over 
the C-space of the robot and are interconnected with a simple, deterministic, and fast planner. We call this planner a connector to emphasize its simplicity (for example, a connector may attempt to connect two configurations by a straight line-segment and fail if this does not lie in the free $\mathrm{C}$-space). For the interconnection, a distance metric is defined in $\mathrm{C}$-space and the connector only tries to connect nodes within a predefined distance. Each successful connection yields an edge of the roadmap. After a large number of nodes have been generated, the "difficult" (narrow) parts of the C-space are identified heuristically and more nodes are placed in these areas [13]. The purpose of this enhancement step is to facilitate the formation of roadmap components that correspond to the components of the free C-space.

A path planning query specifies $a$ and $b$, the initial and the final configurations of the robot. PRM first connects $a$ to a roadmap node by attempting to connect it first to the closest roadmap nodes. Once a connection is obtained, $b$ is tried for connection to the same component. If $A$ and $B$ are the nodes with which $a$ and $b$ get connected respectively, a search of the roadmap can construct a path between $A$ and $B$. The resulting path can be smoothed using any standard smoothing technique.

\subsection{The simplified Probabilistic Roadmap Planner (s-PRM)}

To analyze the performance of the method we work with a simplified algorithm, which we call the simplified Probabilistic Roadmap Planner (s-PRM). We rid the approach of the heuristics employed in practical implementations and any shortcuts taken to achieve better performance.

Initially we assume that the C-space is two-dimensional. Later in the paper, we show that our analysis can be carried over to higher C-space dimensions without any complications.

The parameters of our model are:

\section{- The Free Space}

An arbitrary open subset $\mathcal{F}$ of the unit square $\mathcal{W}=[0,1]^{2}$.

\section{- The Robot}

A point which is free to move in $\mathcal{F}$.

\section{- The Local Connector}

It takes the robot from point $a$ to point $b$ along a straight line and succeeds if the straight 
line segment $\overline{a b}$ is contained in $\mathcal{F}$.

\section{- The Collection of Random Configurations}

A collection of $N$ independent points uniformly distributed over $\mathcal{F}$.

s-PRM works as follows. We throw $N$ independent random points in $\mathcal{F}$ and connect any two of them that can be connected by a free straight line. A roadmap $G$ with possibly more than one connected components results in this fashion. To solve any planning problem, that is to go from any point $a$ to any point $b$, we try to connect both $a$ and $b$ to two nodes in the same connected component of $G$ using straight lines. s-PRM succeeds if and only if this is possible.

Our purpose is to analyze the probability of failure of s-PRM as a function of all the relevant parameters. For this we take any two points $a, b \in \mathcal{F}$, for which we assume that they can be connected via a continuous path

$$
\gamma:[0, L] \longmapsto \mathcal{F}, \text { where } \gamma(0)=a \text { and } \gamma(L)=b \text {. }
$$

(The curve $\gamma$ is parameterized by Euclidean arc length.) Let also $\mathcal{O}$ be the complement of $\mathcal{F}$ in $\mathcal{W}$ (the C-obstacle) and for any $x \in \mathcal{W}$ write $r(x)$ for the Euclidean distance of $x$ to $\mathcal{O}$, that is

$$
r(x)=\inf _{y \in \mathcal{O}}|x-y|
$$

where $|x-y|$ is the Euclidean distance of the points $x$ and $y$ of the plane.

We shall give upper bounds for the probability of failure of s-PRM to find a path from $a$ to $b$. These bounds will involve the number $N$ of random points, the function $r(\gamma(t))$ for $t \in[0, L]$, as well as the length $L$ of $\gamma$, and will hold for any path $\gamma$ that joins $a$ and $b$. The dependence on $L$ and $r(\gamma(t))$ is to be expected. If we have two points $a$ and $b$ for which any connecting path is long, it gets more difficult to find the path, since a larger number of relevant intermediate configurations must be present in our random sample of $\mathcal{F}$. Similarly, if any connecting path has small $r(\gamma(t))$, this intuitively means that the problem is difficult. Imagine for example that $a$ and $b$ are on different sides of narrow passage. The probability of placing random configurations inside the passage and connecting them by straight line paths is small. Let us note here that the quantity $1 / r(\gamma(t))$ has been used as a measure of the "difficulty" of the area in which $\gamma(t)$ lies in the analysis of other planning methods (e.g. [1]). 


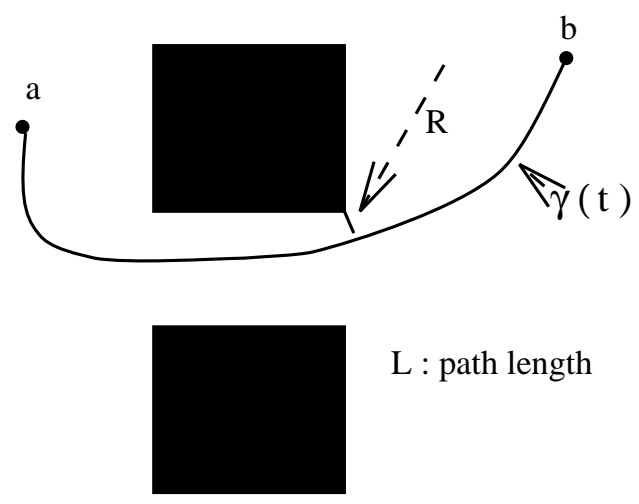

Figure 2: The parameters involved in the theorem

\section{A Bound that Involves the Minimum Distance from the Obstacles}

In this section we derive an upper bound on the failure probability when connecting pairs of points $a$ and $b$. It is assumed that $a$ and $b$ can be connected by some path

$$
\gamma:[0, L] \longmapsto \mathcal{F}
$$

which keeps uniformly away from the obstacles, that is all its points are at least a certain distance $R$ away from the C-obstacles. The key idea is that of covering the path with few balls which overlap to a certain degree. The parameters mentioned in the theorem below are drawn in Figure 2. (Here we follow closely [12].)

Theorem 1 Let $\gamma:[0, L] \longmapsto \mathcal{F}$ be a path of (Euclidean) length $L$, with $\gamma(0)=a, \gamma(L)=b$, and let $R=\inf _{0 \leq t \leq L} r(\gamma(t))$ be the distance of the path to the obstacles.

Then the probability that s-PRM will fail to connect the points a and $b$ is at most

$$
\frac{2 L}{R}\left(1-\alpha R^{2}\right)^{N}
$$

where $\alpha=\pi /(4|\mathcal{F}|)$ is a constant.

Notation: We denote by $d(s, t)$ the distance of the points $\gamma(s)$ and $\gamma(t)$ along the curve $\gamma$. Since we assume that the curve $\gamma$ is parameterized by arc length $d(s, t)=|s-t|$. The ball centered at $x \in \mathbb{R}^{2}$ and with radius $r$ is denoted by $B_{r}(x)$. 


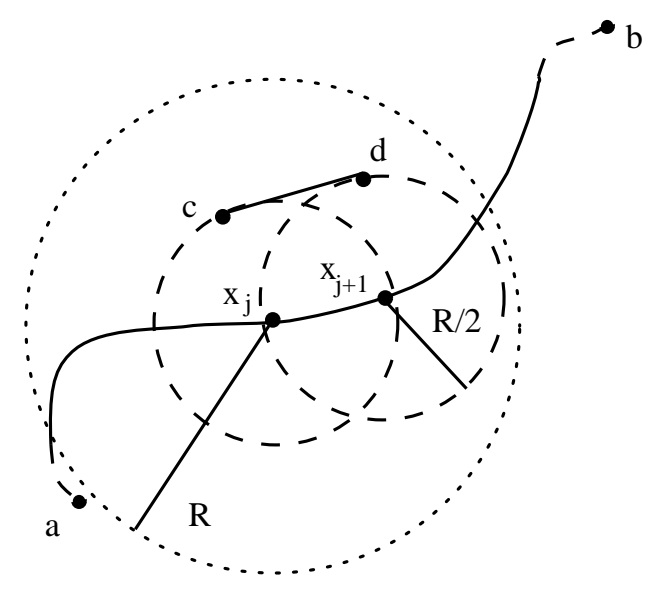

Figure 3: Proof of Theorem 1

Proof: Let $n=\lceil 2 L / R\rceil$. Then we can find points $x_{0}=a, x_{1}, \ldots, x_{n}=b$ on the curve $\gamma$, for which $d\left(x_{j}, x_{j+1}\right) \leq R / 2$, for all $j$. Notice then that

$$
B_{R / 2}\left(x_{j+1}\right) \subseteq B_{R}\left(x_{j}\right), \text { for } j=0, \ldots, n-1 .
$$

This is a direct consequence of the triangle inequality and the inequality $|\gamma(s)-\gamma(t)| \leq d(s, t)$.

Assume now that $c \in B_{R / 2}\left(x_{j}\right)$ and $d \in B_{R / 2}\left(x_{j+1}\right)$. Observe then that also $d \in B_{R}\left(x_{j}\right)$ because of (2). This implies that the straight line segment $\overline{c d}$ is free, since both $c$ and $d$ are contained in the same free ball $B_{R}\left(x_{j}\right)$. An illustration of this basic fact is given in Figure 3 .

Let now $q_{1}, \ldots, q_{N}$ be the random points that our algorithm produced. According to the preceding observation, it is enough to have at least one of the $q_{k}$ 's, $k=1, \ldots, N$, in each ball $B_{R / 2}\left(x_{j}\right), j=1, \ldots, n-1$, for our algorithm to succeed to connect the points $a$ and $b$. Since the $q_{k}$ 's are independent and uniformly distributed over $\mathcal{F}$, we conclude that the probability that the ball $B_{R / 2}\left(x_{j}\right)$ contains none of the $q_{k}$ 's is equal to $\left(1-\left|B_{R / 2}\right| /|\mathcal{F}|\right)^{N}$, where $\left|B_{R / 2}\right|$ is the area of the ball of radius $R / 2$. Here we use the fact that we have thrown $N$ independent points in $\mathcal{F}$.

$$
\begin{aligned}
\operatorname{Pr}[\text { FAILURE }] & \leq \operatorname{Pr}[\text { Some ball is empty] } \\
& \leq \sum_{j=1}^{n-1} \operatorname{Pr}[\text { The } j \text {-th ball is empty }] \\
& =\left(\left\lceil\frac{2 L}{R}\right\rceil-1\right)\left(1-\frac{\left|B_{R / 2}\right|}{|\mathcal{F}|}\right)^{N}
\end{aligned}
$$


But since in two dimensions the area of the ball with radius $R / 2$ is $\pi R^{2} / 4$, the above relation becomes

$$
\operatorname{Pr}[\text { FAILURE }] \leq \frac{2 L}{R}\left(1-\frac{\pi R^{2}}{4|\mathcal{F}|}\right)^{N},
$$

which concludes the proof of the theorem.

\section{A Bound that Exploits Varying Distance}

The analysis of Section 3 uses only the minimum distance of the path $\gamma$ from the obstacles. Yet, if this minimum is achieved rarely, one expects the bound of Theorem 1 to be far from the truth. In this section we establish an upper bound for the failure probability that involves a "mean" distance from the obstacles.

Theorem 2 Let the points $a, b \in \mathcal{F}$ be connected by a path $\gamma:[0, L] \rightarrow \mathcal{F}$, of Euclidean length $L$, and write

$$
r(t)=\inf _{x \in \mathcal{O}}|\gamma(t)-x|
$$

for the distance of $\gamma(t)$ from the obstacles.

Then the probability of failure of s-PRM is at most

$$
6 \int_{0}^{L} \frac{\left(1-(\alpha / 4) r^{2}(t)\right)^{N}}{r(t)} d t
$$

where $\alpha$ is again $\pi /(4|\mathcal{F}|)$.

The idea of the proof is, as was the case in Section 3, to cover the curve $\gamma$ with not-too-many balls that overlap to a certain extent.

Proof: Define $t_{0}=0, r_{0}=r(0)$, and for $k \geq 0$ define

$$
\begin{aligned}
& t_{k+1}=\sup \left\{t \in\left[t_{k}, L\right]: t-t_{k} \leq r_{k}-\frac{1}{2} r(t)\right\} \\
& r_{k+1}=r\left(t_{k+1}\right)
\end{aligned}
$$

and let $n$ be defined by the requirement that $t_{n}=L$. Figure 4 illustrates the selection of points along the path $\gamma$. The above relations guarantee that $\gamma\left(t_{k+1}\right)$ will be chosen as far away from $\gamma\left(t_{k}\right)$ as possible while ensuring that

$$
B_{r_{k+1} / 2}\left(\gamma\left(t_{k+1}\right)\right) \subseteq B_{r_{k}}\left(\gamma\left(t_{k}\right)\right)
$$




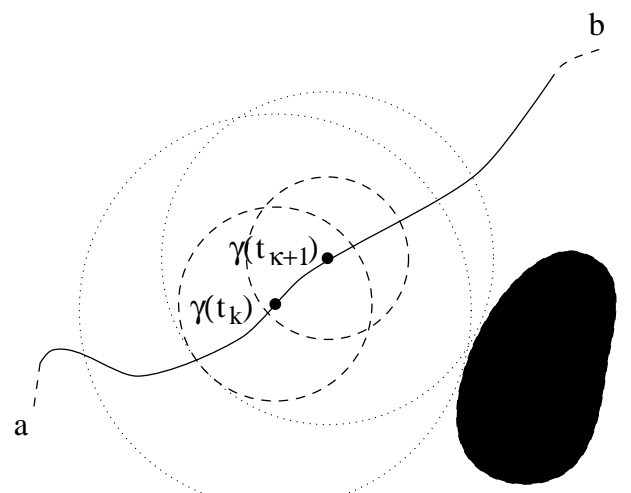

Figure 4: Proof of Theorem 2

for $k=0, \ldots, n-1$. By the same reasoning as in the proof of Theorem 1 ,

$$
\operatorname{Pr}[\text { FAILURE }] \leq \sum_{k=1}^{n-1}\left(1-\alpha r_{k}^{2}\right)^{N} .
$$

Define now the integral

$$
I=\int_{0}^{L} \frac{\left(1-(\alpha / 4) r^{2}(t)\right)^{N}}{r(t)} d t .
$$

Let also

$$
\bar{r}_{k}=\sup _{t_{k} \leq t \leq t_{k}+1} r(t), \quad k=0, \ldots, n-1 .
$$

The function $\left(1-(\alpha / 4) r^{2}\right)^{N} / r$ is a decreasing function of $r$. In each interval $\left[t_{k}, t_{k+1}\right], k=$ $0, \ldots, n-1$, we estimate it from below by its infimum $\left(1-(\alpha / 4) \bar{r}_{k}^{2}\right)^{N} / \bar{r}_{k}$. We thus bound $I$ from below with the corresponding lower Riemann sum

$$
I \geq \sum_{k=0}^{n-1}\left(t_{k+1}-t_{k}\right) \frac{\left(1-(\alpha / 4) \bar{r}_{k}^{2}\right)^{N}}{\bar{r}_{k}} .
$$

Claim 1: $\bar{r}_{k} \leq 2 r_{k}$.

This follows immediately from (5) since, if $\bar{r}_{k}=r\left(\bar{t}_{k}\right)$ for some $\bar{t}_{k} \in\left[t_{k}, t_{k+1}\right]$, we have

$$
r_{k}-\frac{1}{2} \bar{r}_{k} \geq \bar{t}_{k}-t_{k} \geq 0 .
$$

Using Claim 1 equation (8) gives

$$
I \geq \frac{1}{2} \sum_{k=0}^{n-1} \frac{t_{k+1}-t_{k}}{r_{k}}\left(1-\alpha r_{k}^{2}\right)^{N} .
$$


Claim 2: $\forall s, t:|r(s)-r(t)| \leq|s-t|$.

That is, the function $r(t)$ is Lipschitz with Lipschitz constant equal to 1 . This is immediate from the triangle inequality (remember that $\gamma(t)$ has been parameterized by arc length) which gives

$$
r(s) \leq|s-t|+r(t)
$$

and

$$
r(t) \leq|s-t|+r(s)
$$

Claim 3: $\forall k: t_{k+1}-t_{k} \geq \frac{1}{3} r_{k}$.

To see this notice that, by definition, we have

$$
\begin{aligned}
t_{k+1}-t_{k} & =r_{k}-\frac{1}{2} r_{k+1} \\
& \geq r_{k}-\frac{1}{2}\left(r_{k}+\left(t_{k+1}-t_{k}\right)\right)(\text { Claim 2) } \\
& =\frac{1}{2} r_{k}-\frac{1}{2}\left(t_{k+1}-t_{k}\right)
\end{aligned}
$$

which implies $\frac{3}{2}\left(t_{k+1}-t_{k}\right) \geq \frac{1}{2} r_{k}$ and the claim.

Now (9), (7), and Claim 3 give

$$
I \geq \frac{1}{6} \sum_{k=1}^{n-1}\left(1-\alpha r_{k}^{2}\right)^{N} \geq \frac{1}{6} \operatorname{Pr}[\text { FAILURE] }
$$

which concludes the proof of the theorem.

\section{$5 \quad$ Simplified Expressions}

Using the inequality

$$
1-x \leq e^{-x}, \text { for } x \geq 0
$$

we get, from Theorems 1 and 2, the following easier-to-use upper bounds for the failure probability.

- The bound of Theorem 1 becomes

$$
\operatorname{Pr}[\text { FAILURE }] \leq \frac{2 L}{R} \exp \left(-\alpha R^{2} N\right)
$$




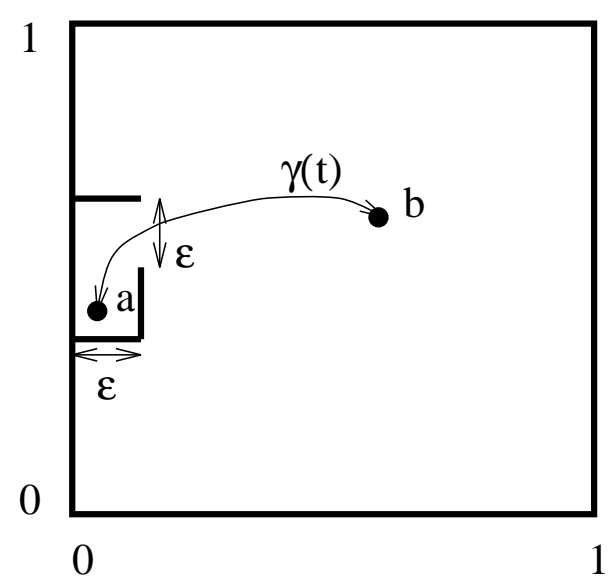

Figure 5: A particular problem

- The bound of Theorem 2 becomes

$$
\operatorname{Pr}[\text { FAILURE }] \leq 6 \int_{0}^{L} \frac{\exp \left(-(\alpha / 4) r^{2}(t) N\right)}{r(t)} d t,
$$

In both formulas above $\alpha=\pi /(4|\mathcal{F}|)$.

\section{Analysis of a Particular Problem}

We have found simple upper bounds for the probability of failure to find a given path with the probabilistic roadmaps method. We are now going to use these bounds to derive estimates on $N$, the number of random points thrown uniformly in the free $\mathrm{C}$-space, in order to have the probability of failure less than a respecified number, say, for the sake of the argument, less than $1 / 2$.

In this section we study a simple problem in two dimensions. For the problem shown in Figure 5 we estimate $N$ using equation (10), equation (11) and the method of analysis of [15].

The parameter of the problem is $\epsilon$, the length of the opening near point $a$, which is taken to tend to 0 .

We have $|\mathcal{F}|=1$ so that $\alpha=\pi / 4$. We also have $L \asymp 1$ and $R \asymp \epsilon$. (By $x \asymp y$ we mean that $C^{-1} x \leq y \leq C x$, for some absolute constant $C>0$. In what follows $C$ stands for an absolute positive constant, not necessarily the same in all its occurrences. However, it is always possible to find a definite number for every occurrence of $C$.) 


\section{Estimate using (10):}

We get

$$
\begin{aligned}
\operatorname{Pr}[\text { FAILURE }] & \leq \frac{2 L}{R} \exp \left(-\alpha R^{2} N\right) \\
& \leq C \frac{1}{\epsilon} \exp \left(-C \epsilon^{2} N\right)
\end{aligned}
$$

If we choose

$$
N \asymp \frac{1}{\epsilon^{2}} \log \frac{1}{\epsilon}
$$

we achieve that the failure probability is bounded above by a small constant (which, of course, depends on the constant implied by the $\asymp \operatorname{sign}$ in (12), but it is the dependence of $N$ on $\epsilon$ that we care about here).

\section{Estimate using (11):}

The function $r(t)$ for the path of Figure 5 clearly satisfies

$$
r(t) \asymp\left\{\begin{array}{ll}
\epsilon & \text { if } 0 \leq t \leq C \epsilon \\
t & \text { if } t \geq C \epsilon
\end{array} .\right.
$$

Equation (11) then gives

$$
\begin{aligned}
\operatorname{Pr}[\text { FAILURE }] & \leq 6 \int_{0}^{L} \frac{\exp \left(-(\alpha / 4) r^{2}(t) N\right)}{r(t)} d t \\
& \leq C \exp \left(-C \epsilon^{2} N\right) \int_{0}^{L} \frac{d t}{r(t)}
\end{aligned}
$$

and

$$
\begin{aligned}
\int_{0}^{L} \frac{d t}{r(t)} & \leq \int_{0}^{C \epsilon} \frac{d t}{r(t)}+C \int_{C \epsilon}^{L} \frac{d t}{t} \\
& \asymp C \epsilon \frac{1}{\epsilon}+\log \frac{1}{\epsilon} \asymp \log \frac{1}{\epsilon}
\end{aligned}
$$

Therefore

$$
\operatorname{Pr}[\text { FAILURE }] \leq C \exp \left(-C \epsilon^{2} N\right) \log \frac{1}{\epsilon}
$$

and choosing

$$
N \asymp \frac{1}{\epsilon^{2}} \log \log \frac{1}{\epsilon}
$$


bounds the failure probability from above by a small constant.

\section{Estimate after [15]:}

The space in Figure 5 is, in the terminology of [15], a $\left(C \epsilon^{2}\right)$-good space. This means that every point of $\mathcal{F}$ can be connected with a free straight line segment to a set of points of $\mathcal{F}$ whose area is at least $C \epsilon^{2}$ (clearly because of the box on the left).

Then (see Theorem 2.1 in [15] and the definition of adequate sets of points) one needs to have

$$
N \asymp \frac{1}{\epsilon^{2}} \log \frac{1}{\epsilon}
$$

in order to bound the failure probability away from 1.

\section{Comparison:}

Theorem 2 clearly exploits the fact that $r(t)$ is small only briefly to gain an extra logarithm in the estimate (13) with respect to (12) and (14). Note that Theorem 1 and the approach taken in [15] refer to quantities - the minimum of $r(t)$ and the goodness of $\mathcal{F}$ - which are single numbers defined globally over the whole space. Theorem 2 achieves a better estimate for $N$ because it is less sensitive to local difficulties than are the bounds of Theorem 1 and the analysis in [15].

It should be said that the true answer is easily seen to be

$$
N \asymp \frac{1}{\epsilon^{2}},
$$

since it is necessary and sufficient to put a bounded number of points in the box, which happens with probability $\asymp \epsilon^{2}$. The estimates (12), (13) and (14) can thus be seen as the unavoidable $1 / \epsilon^{2}$ times a factor on which they are to be compared. According to (11) and after estimating (from above) the exponential by $\exp \left(-(\alpha / 2) R^{2} N\right)$ (remember $R=\inf _{t} r(t)$ ) that factor takes the very simple form

$$
\frac{L}{R_{h}}=\int_{0}^{L} \frac{d t}{r(t)}
$$

where $R_{h}$ is the harmonic mean of the function $r(t)$. 


\section{Higher Dimension}

Everything in the preceding analysis extends without any changes to spaces of higher dimension. Let $d$ be the dimension of the space and $\omega_{d}$ denote the $d$-dimensional volume of the unit ball in $\mathbb{R}^{d}$. Let also $\alpha_{d}=2^{-d} \omega_{d} /|\mathcal{F}|$.

- The bound corresponding to (10) becomes then

$$
\operatorname{Pr}[\text { FAILURE }] \leq \frac{2 L}{R} \exp \left(-\alpha_{d} R^{d} N\right)
$$

- The bound corresponding to (11) becomes

$$
\operatorname{Pr}[\text { FAILURE }] \leq 6 \int_{0}^{L} \frac{\exp \left(-\alpha_{d} 2^{-d} r^{d}(t) N\right)}{r(t)} d t
$$

\section{Discussion}

The bounds computed in this paper are not very easy to use since they depend on the properties of the postulated connecting path $\gamma(t)$ from $a$ to $b$, which are difficult to measure a priori. Nevertheless, they at least shed light on the nature of the dependence of the algorithm on these properties. The fact that the dependence on $N$ is exponential is a good and, of course, expected feature. Another nice feature revealed is that the dependence on $L$ is linear. The bound of Theorem 2 that exploits varying distance from the obstacles makes our analysis useful in spaces where there are narrow regions and large parts of free $\mathrm{C}$-space. This analysis also emphasizes the distinguishing features of PRM when compared with a planner that creates a uniform grid of points of appropriate resolution in the free $\mathrm{C}$-space. The latter planner places many nodes in large free parts of the C-space while a probabilistic technique like PRM as described in [13] will avoid creating a large number of nodes in areas where local connections are obtained easily. Undoubtedly, this contributes to the good experimental performance of PRM.

What the bounds given by Theorems 1 and 2 and by the simplified inequalities (10) and (11) do permit us to do is to answer questions of the type: "Assuming that there is a path from a to $b$ which stays away from the obstacles a distance at least $\epsilon$, what should $N$ be in order to guarantee a success probability of at least 0.99 ?" Or, if we know the optimal path from $a$ to $b$ and this stays $\epsilon$-away from the obstacles, we can estimate what $N$ should be in order to find, with a predefined 
probability, a path which stays $\eta$-close $(\eta<\epsilon)$ to the optimal path. This is done simply by using $\eta$ as the distance of the optimal path from the obstacles.

We have thus obtained quite workable expressions for the failure probability and have demonstrated their use with a simple but illuminating example. It should also be clarified that, in practice, one need not restrict oneself to using the Euclidean distance. No special properties of it were used in this paper and any other distance would give analogous results. Finally, we note that probabilistic roadmaps have been applied successfully to non-holonomic robots and the interested reader is referred to [21] for the analysis in that case.

It is admittedly very hard to characterize the average performance of PRM and we view the work in this paper as complementary to the work in [15] which identified a property of the C-space, called $\epsilon$-goodness, that is likely to account for the success of PRM in real settings. Our analysis relates in direct way certain geometric properties of the configuration space of the robot to the parameters of the planning algorithm used. We are confident that research along the direction of understanding how the geometric properties of the environment of the robot influence the performance of specific planning algorithms will be very important in the future and can guide us to design faster planners.

Acknowledgments: L. Kavraki and J.-C. Latombe were partially supported by ARPA grant N00014-94-1-0721. M. Kolountzakis was supported by NSF grant DMS-9304580. The authors would like to thank the anonymous reviewers for their comments.

\section{References}

[1] J.M. Ahuactzin Larios, Le Fil d'Ariane: Une Méthode de Planification Générale. Application à la Planification Automatique de Trajectoires, Thèse de l'Institut Nat. Polytechnique de Grenoble, September 1994.

[2] J. Barraquand and P. Ferbach, "Path planning through variational dynamic programming", Proc. of IEEE ICRA, 1839-1846, San Diego, CA, 1994.

[3] J. Barraquand and J.-C. Latombe, "Robot motion planning: A distributed representation approach", Intl. J. of Rob. Res., 10:628-649, 1991. 
[4] J. Barraquand, L. Kavraki, J.-C. Latombe, T.-Y. Li, R. Motwani, and P. Raghavan, "A Random Sampling Scheme for Motion Planning", Robotics Research, G. Giralt and G. Hirzinger, North Holland, 1996.

[5] P. Bessierre, E. Mazer, and J.-M. Ahuactzin, "Planning in a continuous space with forbidden regions: the Ariadne's clew algorithm", Algorithmic Foundations of Robotics, K. Goldberg et al. (eds.), A K Peters, Wellesley, MA, 1995.

[6] H. Chang, and Tsai-Yen Li, "Assembly maintainability study with motion planning", Proc. of IEEE ICRA, 1012-1019, Nagoya, Japan, 1995.

[7] P.C. Chen, "Learning to improve path planning performance", Report SAND95-0722, Sandia National Laboratories, 1995.

[8] D. Challou and M. Gini, "Parallel Formulation of Informed Randomized Search for Robot Motion Planning Problems", Proc. of IEEE ICRA, 709-714, Nayoga, Japan, 1995.

[9] K. Gupta and X. Zhu, " Practical motion planning for many degrees of freedom: A novel approach within sequential framework", Proc. of IEEE ICRA, 2038-2043, San Diego, CA, 1994.

[10] Y.K. Hwang and C. Chen, "A heuristic and complete planner for the classical mover's problem", Proc. of IEEE ICRA, 729-736, Nagoya, Japan, 1995.

[11] Th. Horsch, F. Schwarz, and H. Tolle, "Motion planning for many degrees of freedom random reflections at C-space obstacles" Proc. IEEE ICRA, 3318-3323, San Diego, CA, 1994.

[12] L. Kavraki, Random networks in configuration space for fast path planning, Ph.D. Thesis, STAN-CS-TR-95-1535, January 1995.

[13] L. Kavraki, J.-C. Latombe, "Randomized preprocessing of configuration space for fast path planning", Proc. IEEE ICRA, 2138-2145, San Diego, CA, 1994.

[14] L. Kavraki, P. Švestka, J.-C. Latombe and M. Overmars, "Probabilistic roadmaps for fast path planning in high dimensional configuration spaces", to appear in IEEE Tr. on Robotics and Automation; also STAN-CS-TR-94-1519. 
[15] L. Kavraki, J.-C. Latombe, R. Motwani, and P. Raghavan, "Randomized query processing in robot path planning", 27th Annual ACM Symposium on Theory of Computing (STOC), 353-362, Las Vegas, NV, 1995.

[16] K. Kondo, "Motion planning with six degrees of freedom by multistratergic bidirectional heuristic free space enumeration", IEEE Tr. on Rob. and Autom., 7(3):267-277, 1991.

[17] F. Lamiraux and J.P. Laumond, "On the expected complexity of random path planning", Centre National de la Recherche Scientifique, LAAS Report No 95087, March 1995.

[18] J.-C. Latombe, Robot Motion Planning, Kluwer Academic Publishers, Boston, 1991.

[19] T. Lozano-Pérez, "Spatial planning: A configuration space approach", IEEE Tr. on Computers, 32:108-120, 1983.

[20] M. Overmars and P. Švestka, "A probabilistic learning approach to motion planning" Algorithmic Foundations of Robotics, K. Goldberg et al. (eds.), A K Peters, Wellesley, MA, $19-37,1995$.

[21] P. Švestka and M. Overmars "Motion planning for car-like robots using probabilistic roadmps", RUU-CS-94-33, Comp. Sci., Utrecht Univ., the Netherlands, 1994. 